\title{
Sensitivity of unsteady collapsible channel flows to modelling assumptions
}

\author{
H. F. Liu ${ }^{1}$, X. Y. Luo ${ }^{1, *, \dagger}$, Z. X. Cai ${ }^{2}$ and T. J. Pedley ${ }^{3}$ \\ ${ }^{1}$ Department of Mathematics, University of Glasgow, Glasgow G12 8QW, U.K. \\ ${ }^{2}$ Department of Mechanics, Tianjin University, Tianjin, China \\ ${ }^{3}$ DAMTP, University of Cambridge, Cambridge CB3 OWA, U.K.
}

\begin{abstract}
SUMMARY
We investigate the influences of modelling assumptions on the dynamic behaviour of collapsible channel flows. The elastic wall is modelled in various different ways: as a large strain Bernoulli-Euler beam, as a small strain Timoshenko beam, and as a 2D-solid model derived from a general virtual work approach, using small strain or large strain assumptions. Different inlet boundary conditions are also considered. The in-house finite element codes and the commercial finite element package ADINA 8.4 are used. The steady results agree very well when using the different models/approaches. The unsteady results, on the other hand, can be quite different. The dynamic behaviour of the system is analysed for a set of chosen parameters, using the full numerical solvers, the linear stability analysis, and the Fourier transform. It is found that the system stability is highly sensitive to the solid modelling assumptions used, numerical solvers adopted, or the boundary conditions imposed. Accuracy of the numerical schemes also has an impact on the system's unsteady behaviour. However, despite the high sensitivity of the unsteady solutions to the modelling assumptions, a cascade stability structure previously revealed by the authors seems to exist when different numerical approaches are used. Copyright (C) 2009 John Wiley \& Sons, Ltd.
\end{abstract}

Received 24 July 2008; Revised 14 November 2008; Accepted 24 November 2008

KEY WORDS: collapsible channel flow; self-excited oscillations; cascade structure; fluid-structure interactions; linear stability analysis; finite element methods

\section{INTRODUCTION}

For many researchers, the motivation of studying flow in collapsible tubes originates not only from the potential applications to various biomedical problems, but perhaps more from academic

\footnotetext{
*Correspondence to: X. Y. Luo, Department of Mathematics, University of Glasgow, Glasgow G12 8QW, U.K.

${ }^{\dagger}$ E-mail: x.y.luo@maths.gla.ac.uk

Contract/grant sponsor: U.K. Royal Society of London

Contract/grant sponsor: U.K. Royal Academy of Engineering

Contract/grant sponsor: Tianjin Municipal Science and Technology Commission
} 
curiosity about the rich dynamic behaviour revealed by the experiments [1-3], such as flow limitation and self-excited oscillations. Early work includes the simplified 'lumped-parameter' and 1D models $[4,5]$, followed by $2 \mathrm{D}$ models in which the collapsible tube was simplified as a thin membrane [6-10]. In these studies, the major findings are that the self-excited oscillations are caused by the instabilities of the system, and that the energy dissipation plays a key role in the instabilities. Multiple modes of oscillation of different frequencies are observed in the simulations, which bear a close resemblance to those observed in experiments (e.g. [2]).

Recently, the elastic wall model has been improved by using the Bernoulli-Euler or Timoshenko beam $[11,12]$, which demonstrates the effects of the bending stiffness and the significant role of the 'effective tension'. Work has also begun to extend the study to 3D modelling [13-16]. However, due to the high complexity of the problem and the limitation of computational resources, to date these studies have been limited to steady, or buckling analysis, or high-frequency small deformation cases.

Using a 2D fluid-beam model (FBM), in which the solid equilibrium equations of the elastic wall can be satisfied in both normal and tangential directions, Luo et al. [17] identified a cascade structure of the linear stability of the system in the wall stiffness and Reynolds number parameter space. However, given the rich dynamics of the system, it is not clear if this cascade structure exists only under the specific modelling assumptions employed by the FBM, and how sensitive the system stability and the self-excited oscillations are to different numerical approaches.

The focus of this study is to test the influence of various modelling assumptions on the dynamic behaviour of the collapsible channel flow. This is done by carefully analysing both steady and unsteady results of three different cases, A, B, C, where the solutions can be either stable or unstable. In addition to FBM, ADINA 8.4, the commercial finite element package, which can model more general fluid-structure interaction (FSI) problems [18], is used to simulate the unsteady behaviour of the system with different modelling assumptions. The results are then compared with those from the FBM model and its eigenvalue solver. Different inlet boundary conditions, i.e. whether the flow rate or pressure is specified, are also examined.

\section{MODELLING}

The numerical model consists of a steady flow in a channel in which part of the upper wall is replaced by an elastic beam in the plane strain configuration; see Figure 1. The rigid channel has width $\mathrm{D}$, a part of the upper wall of length $\mathrm{L}$ is replaced by a pre-stressed elastic beam subjected to an external pressure $p_{\mathrm{e}}$, and $L_{\mathrm{u}}$ and $L_{\mathrm{d}}$ are the lengths of the upstream and downstream rigid parts of the channel. Steady Poiseuille flow with average velocity $U_{0}$ is assumed at the entrance. The flow is incompressible and laminar, with the fluid having density $\rho$ and viscosity $\mu$. The extensional and bending stiffnesses of the beam are $E A$ and $E J$, respectively, where $E$ is Young's modulus, ${ }^{\ddagger} A$ is the cross-sectional area of the beam, which is equivalent to the beam's thickness, $h$, as the beam has a unit width in the $z$-direction, and $J$ is the moment of inertia of the beam cross-section. The pre-tension in the beam is assumed to be zero, and the density, damping, and rotational inertia of the beam are all neglected.

\footnotetext{
${ }^{\ddagger}$ As we consider a plane strain problem here, $E$ is equivalent to the conventional Young’s modulus $E_{A}$ divided by $\left(1-v^{2}\right)$, where $v$ is Poisson's ratio.
} 


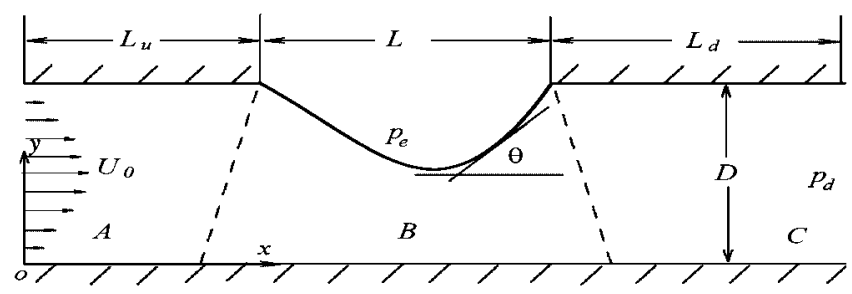

Figure 1. The flow-beam configuration (not to scale). Part B has part of the wall being replaced by an elastic beam.

\subsection{The FBM}

2.1.1. Governing equations. For convenience, the flow velocity components $u_{i}$, fluid stresses $\sigma_{i j}$, pressure $p$, time $t$, the Cartesian coordinates $x, y$ (with origin at the left bottom of the channel), and length $l$ are non-dimensionlized as follows:

$$
u_{i}^{*}=\frac{u_{i}}{U_{0}}, \quad \sigma_{i j}^{*}=\frac{\sigma_{i j}}{\rho U_{0}^{2}}, \quad p^{*}=\frac{p}{\rho U_{0}^{2}}, \quad t^{*}=\frac{t U_{0}}{D}, \quad l^{*}=\frac{l}{D}, \quad x^{*}=\frac{x}{D}, \quad y^{*}=\frac{y}{D} \quad(i=1,2)
$$

and the non-dimensional parameters, such as the curvature $\kappa$, the wall stiffness $c_{\lambda}, c_{\kappa}$, and the Reynolds number are defined by

$$
\kappa^{*}=\kappa D, \quad c_{\lambda}=\frac{E A}{\rho U_{0}^{2} D}, \quad c_{\kappa}=\frac{E J}{\rho U_{0}^{2} D^{3}}, \quad R e=\frac{U_{0} D \rho}{\mu}
$$

The variables with a star are the non-dimensional ones, which will be used throughout this paper. In the following, however, the stars are dropped for simplicity.

The governing equations for the fluid flow are the Navier-Stokes equations

$$
\begin{aligned}
\frac{\partial u_{i}}{\partial t}+u_{j} u_{i, j} & =-p_{, i}+\frac{1}{R e} u_{i, j j} \\
u_{i, i} & =0, \quad i, j=1,2
\end{aligned}
$$

By employing the Kirchhoff constitutive laws for the elastic beam, we can write the following dimensionless governing equations for the beam (for derivation of these equations, see Cai and Luo [11]

$$
\begin{aligned}
c_{\kappa} \kappa \kappa^{\prime}+c_{\lambda} \lambda^{\prime} & =-\lambda \tau_{\mathrm{n}} \\
c_{\kappa}\left(\frac{1}{\lambda} \kappa^{\prime}\right)^{\prime}-c_{\lambda} \lambda \kappa(\lambda-1) & =\lambda\left(\sigma_{\mathrm{n}}-p_{\mathrm{e}}\right) \\
x^{\prime}=\lambda \cos \theta, \quad y^{\prime} & =\lambda \sin \theta, \quad \theta^{\prime}=\lambda \kappa
\end{aligned}
$$

In the above expressions, the superscript prime stands for a derivative with respect to the initial beam position $l$, and $\lambda$ is the principal stretch of the beam, defined here as

$$
\lambda=\sqrt{x^{\prime 2}+y^{\prime 2}}
$$


$\theta$ stands for the rotation angle of the beam from the $x$-axis; $\sigma_{\mathrm{n}}, \tau_{\mathrm{n}}$ are fluid stress applied on the beam in the normal and tangential direction, respectively

$$
\sigma_{\mathrm{n}}=p-\frac{2}{R e} \frac{\partial u_{n}}{\partial n}, \quad \tau_{\mathrm{n}}=-\frac{1}{R e}\left(\frac{\partial u_{s}}{\partial n}+\frac{\partial u_{n}}{\partial s}\right)
$$

where $n$ and $s$ denote the normal and tangential direction of the beam. Note $c_{\kappa}$ and $c_{\lambda}$ are related by $c_{\kappa} / c_{\lambda}=\left(h^{2} / 12 D^{2}\right) \approx 10^{-5}$ if the beam thickness is $1 \%$ of the channel height.

Equation (4) is the momentum balance in the tangential direction: the left-hand terms are the bending-induced tangential force $c_{\kappa} \kappa \kappa^{\prime}$ and the stretching-induced tangential force $c_{\lambda} \lambda^{\prime}$; the righthand term is the external tangential force due to the fluid $\lambda \tau_{n}$. Equation (5) gives the normal momentum balance: the left-hand terms are the bending-induced normal force $c_{\kappa}\left(1 / \lambda \kappa^{\prime}\right)^{\prime}$ and the stretching-induced normal force (tension) $c_{\lambda} \lambda \kappa(\lambda-1)$, and the right-hand side term is proportional to the transmural pressure, $\left(\sigma_{\mathrm{n}}-p_{\mathrm{e}}\right)$.

It is worth mentioning that in deriving Equations (4)-(7), we have not made the small strain assumption for the beam, which implies that the problem is suitable for a large strain problem as long as the constitutive equation is satisfied. However, we have assumed that both the wall inertia and initial tension are zero in this study.

The boundary condition is set up in such a way that no-slip boundary condition is applied at the fluid-structure interface; and the two ends of the elastic wall are clamped. A constant external $p_{\mathrm{e}}$ is applied above the elastic section, and a stress free condition is used for fluid $\sigma_{\mathrm{nd}}\left(\approx p_{\mathrm{d}}\right)=0$. This also determines the transmural pressure at the downstream end: $\sigma_{\text {nd }}-p_{\mathrm{e}}\left(\approx p_{\mathrm{d}}-p_{\mathrm{e}}\right)=-p_{\mathrm{e}}$.

In all but Section 3.7, a steady parabolic velocity profile is imposed at the inlet with the average velocity $U_{0}$. In Section 3.7 , however, instead of specifying the velocity profile, we specify the pressure drop at the entrance: $p_{\mathrm{u}}-p_{\mathrm{d}}=p_{\mathrm{u}}$. All other conditions remain the same.

2.1.2. Numerical schemes. Two approaches are used to solve the FBM model. One is the fully coupled FSI solver for the unsteady flows and the other is the eigenvalue solver for the linear stability analysis.

2.1.2.1. Fully coupled FSI solver. The finite element code solves the fully coupled unsteady non-linear FSI equations simultaneously. To deal with the FSI procedure, an arbitrary LagrangianEulerian formulation is used. For this purpose, the mesh is divided into three regions: A, B, and $\mathrm{C}$, where $\mathrm{B}$ is the adaptive mesh region under the elastic beam and $\mathrm{A}, \mathrm{C}$ are fixed mesh regions for upstream and downstream parts, respectively. In order to allow for a movable boundary and meanwhile to secure the quality of the mesh in the fluid domain, an adaptive mesh with rotating spines is employed in the elastic region under the beam.

The fluid and beam equations are solved simultaneously with the weighted residual methods. The system equations (2)-(6) are discretized with Petrov-Galerkin method and the fluid elements are triangular 6-node elements with second-order shape function for $u, v$ and linear function for $p$, while beam elements are 3-node elements with second-order shape function for $x, y, \theta, \lambda, \kappa$. The discretized finite element equations can be written in matrix form as

$$
M(U) \frac{\mathrm{d} U}{\mathrm{~d} t}+K(U) U-F=R=0
$$

where $U=\left(u_{j}, v_{j}, p_{j}, x_{j}, y_{j}, \theta_{j}, \lambda_{j}, \kappa_{j}\right)$ is the global vector of unknowns, with $j=1,2, \ldots, n$ the nodal number, and $R$ is the overall residual vector. 
The matrix equation (9) is solved with an implicit finite difference second-order predictorcorrector scheme with variable time steps. Details of the numerical scheme can be found in [11].

2.1.2.2. The eigenvalue solver. We denote the infinitesimal perturbation to a steady solution $\bar{U}$ by $\Delta U$, so that $U=\bar{U}+\Delta U$ is a solution to the system. Here $\bar{U}$ is obtained from a full numerical simulation as described above. If the system is stable, then $U$ approaches $\bar{U}$ as time increases. Writing $\Delta U$ in the form of $\mathrm{e}^{\omega t} \tilde{U}$, where $\omega\left(=\omega_{r}+\mathrm{i} \omega_{i}\right), \tilde{U}$ are the complex eigenvalue and eigenvector, respectively, and substituting into (9), we obtain a matrix eigenvalue equation

$$
(\omega \bar{M}+\bar{K}) \tilde{U}=\tilde{R}=0
$$

where the matrices $\bar{M}, \bar{K}$ are determined by the steady solution $\bar{U}$. The matrix equation (10a) is solved by using a QZ algorithm [19], which solves for the complete set of eigensolutions of the system. The eigensolution can also be expressed in the form

$$
\begin{aligned}
\Delta U & =\operatorname{Re}\left[\mathrm{e}^{\left(\omega_{r}+\mathrm{i} \omega_{i}\right) t}\left(\tilde{U}_{r}+\mathrm{i} \tilde{U}_{i}\right)\right] \\
& =\mathrm{e}^{\omega_{r} t}\left(\tilde{U}_{r} \cos \left(\omega_{i} t\right)-\tilde{U}_{i} \sin \left(\omega_{i} t\right)\right)=\mathrm{e}^{\omega_{r} t}\|\tilde{U}\| \cos \left(\omega_{i} t+\phi\right)
\end{aligned}
$$

where $\|\tilde{U}\|=\sqrt{\tilde{U}_{r}^{2}+\tilde{U}_{i}^{2}}$ is the amplitude and $\phi=\arctan \tilde{U}_{i} / \tilde{U}_{r}$ is the initial phase. This form will be used to present the eigenvalue results later.

\section{2. $A D I N A$}

2.2.1. Governing equations. The same configuration (Figure 1) is solved using ADINA. The basic governing equations for fluids are the same as (1)-(3). However, for the solid, several approaches are possible. For a large strain and large displacement plane strain problem, one may choose to use a general 2D approach derived from the principle of virtual work [20]

$$
\int_{V} \tau_{i j} \delta e_{i j} \mathrm{~d} V=\int_{s} f^{s} \delta u^{s} \mathrm{~d} S, \quad i, j=1,2
$$

where $\tau_{i j}$ is the Cauchy stress tensor, $e_{i j}$ is the strain tensor corresponding to virtual displacements, and $V$ is the volume, $S$ is the surface on which external traction (pressure) $f^{s}$ is applied, and $\delta u^{s}$ are the components of virtual displacement vector evaluated on the surface $S$. For a small strain and large displacement problem, to save computational cost, one can use the iso-beam model based on a Timoshenko beam model [21]. The Timoshenko beam deformation is shown in Figure 2 (again for simplicity, the inertia of the beam is neglected), with the governing equations [20]

$$
\begin{array}{r}
E_{A} A \int_{0}^{L} \frac{\mathrm{d} u}{\mathrm{~d} x} \frac{\mathrm{d} \bar{u}}{\mathrm{~d} x} \mathrm{~d} x=\int_{0}^{L} f \bar{u} \mathrm{~d} x \\
E_{A} J \int_{0}^{L}\left(\frac{\mathrm{d} \beta}{\mathrm{d} x}\right)\left(\frac{\mathrm{d} \bar{\beta}}{\mathrm{d} x}\right) \mathrm{d} x+G A k \int_{0}^{L}\left(\frac{\mathrm{d} w}{\mathrm{~d} x}-\beta\right)\left(\frac{\mathrm{d} \bar{w}}{\mathrm{~d} x}-\beta\right) \mathrm{d} x=\int_{0}^{L} p \bar{w} \mathrm{~d} x
\end{array}
$$

where the overbar denotes the virtual quantities, $\beta(=\mathrm{d} w / \mathrm{d} x-\gamma)$ denotes the rotation angle of the plane section initially normal to the neutral axis, $\mathrm{d} w / \mathrm{d} x$ stands for the total rotation angle of 


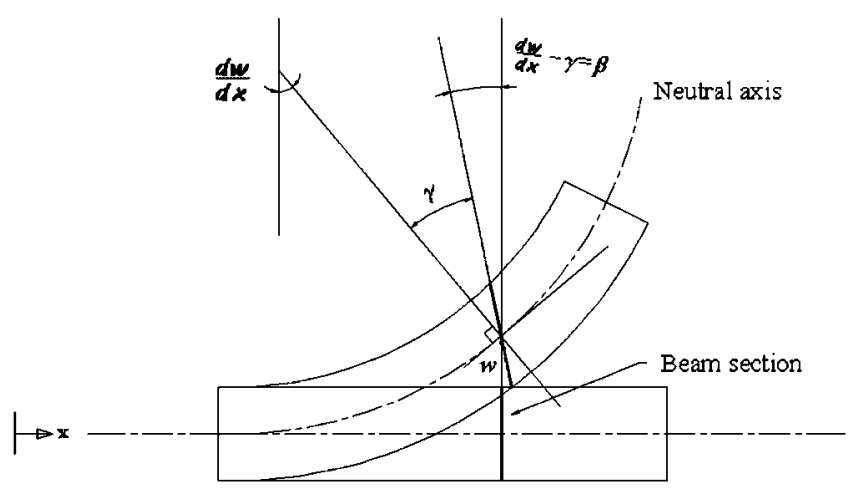

Figure 2. Sketch of the Timoshenko beam deformation.

the beam neutral axis and $\gamma$ is the shearing strain, $G$ is the shear modulus, and $k$ is the ratio of the shear area and the total cross-sectional area $A$, which is estimated to be $\frac{5}{6}$ for a rectangular cross-section beam [22]. The terms on the right-hand side of (12) and (13) are external loading and external moment, caused by the shear stress of the fluid, and the transmural (fluid normal stress minus external) pressure, respectively.

For the small strain formulation, the total Lagrangian formulation is imposed with the second Piola-Kirchhoff stress and Green-Lagrange strain. For the large strain formulation, the updated Lagrangian formulation with Cauchy stress and logarithmic strain is imposed $[18,20]$. For the unsteady simulations, a second-order implicit composite scheme is used for the time integration with a fixed time step [18].

The boundary conditions are taken to be the same as in the FBM model.

2.2.2. Numerical setup in ADINA. The mesh for the fluid domain is also divided into three regions: below the elastic beam, upstream, and downstream. A grid independence test is carried out for three different meshes ranging from coarse to finer grids, namely, $8 \times(35+45+80), 11 \times(46+60+110)$, $17 \times(70+90+165)$. It is found that the results become almost identical for the last two meshes tested. Therefore, the grid for the fluid domain selected for this problem is $11 \times(46+60+110)$.

Two types of elements in ADINA are selected to model the beam

1. 2D solid. These elements are chosen to study unsteady results' sensitivity to the structural assumption (large/small strain). The mesh is discretized with 400 9-node second-order quadrilateral elements.

2. Iso-beam. These elements are only suitable for small strain problem, and are chosen to study the unsteady results' sensitivity to model assumptions. The beam domain is discretized with 200 4-node elements.

Dimensional variables are used in ADINA. These are related to the dimensionless FBM variables in the following way:

$$
U_{0}=\frac{R e \cdot \mu}{\rho \cdot D}, \quad P e_{A}=P e \rho U^{2}, \quad E_{A}=E\left(1-v^{2}\right)=\frac{c_{\lambda} \rho U_{0}^{2} D\left(1-v^{2}\right)}{A}
$$


2.2.3. Numerical schemes used in ADINA. The Galerkin finite element method is employed to discretize the fluid governing equations. The fluid domain was discretized with 9-node quadrilateral finite element which is second order in spatial interpolation. The solution of the coupling structure and fluid system is obtained by adopting a direct approach where the equations for structure and those for fluid are combined into a matrix system of equations and are solved simultaneously. The solution of the highly nonlinear system of equations is then solved using the Newton-Raphson method followed by the Gaussian elimination.

\subsection{Comparison of the beam models used in FBM and ADINA}

Although both solve the same fluid equations, the FBM and ADINA use different configurations for the beam model, which can be compared by analysing their governing equations.

For ADINA's Timoshenko beam (iso-beam), in (12), the left-hand side term is the stretchinduced tangential force, which is similar to the second left-hand side term in (4) of the FBM's modified Bernoulli beam. However, note that the term corresponding to $c_{\kappa} \kappa \kappa^{\prime}$ is ignored in (12). Without this term, (4) and (12) are essentially the same.

In addition, in ADINA, (13) implies that bending and shearing effects are balancing the external force in the direction normal to the beam. In (13), the first term on the left-hand side represents the bending term, similar to the first term on the left-hand side in (5), the second term stands for the shear force term. Whereas for FBM, (5) represents the equilibrium of the internal bending and the induced tension, $\lambda \kappa c_{\lambda}(\lambda-1)$, with the external force $\lambda\left(p_{\mathrm{e}}-\sigma_{\mathrm{n}}\right)$. That is no shear strain contribution was taken into account.

It is easy to show that these two beam models are the same if ADINA does not include the shear strain term in (13) and FBM (5) does not include the coupling terms between the stretch and bending deformation, i.e. $c_{\kappa} \kappa \kappa^{\prime}$ and $\lambda \kappa c_{\lambda}(\lambda-1)$. The bending term in $\operatorname{FBM} c_{\kappa}\left((1 / \lambda) \kappa^{\prime}\right)^{\prime}$ can be written in a dimensional way as $E J\left(\kappa^{\prime \prime}\right)$. From (6), $\kappa=\mathrm{d} \theta / \mathrm{d} s$, and for small deformation, we can consider only the vertical deformation, i.e. $s=x$ and $\theta=\mathrm{d} w / \mathrm{d} x$; therefore, the first left-hand side term in (5) becomes

$$
E J\left(\kappa^{\prime \prime}\right)=E J \frac{\mathrm{d}^{4} w}{\mathrm{~d} x^{4}}
$$

The first left-hand term in (13) can be written as

$$
E J \int_{0}^{L} \frac{\mathrm{d} \beta}{\mathrm{d} x} \frac{\mathrm{d} \bar{\beta}}{\mathrm{d} x} \mathrm{~d} x=E J \int_{0}^{L} \frac{\mathrm{d}^{2} w}{\mathrm{~d} x^{2}} \frac{\mathrm{d}^{2} \bar{w}}{\mathrm{~d} x^{2}} \mathrm{~d} x=E J \int_{0}^{L} \frac{\mathrm{d}^{4} w}{\mathrm{~d} x^{4}} \frac{\mathrm{d} \bar{w}}{\mathrm{~d} x} \mathrm{~d} x
$$

Since (15) and (16) are identical (i.e. FBM, (5) and ADINA, (13) are identical under these conditions), we conclude that FBM without the coupling of the stretching and bending terms is the same as ADINA's Timoshenko beam without the shear strain.

Note that for the case of short and thick beam and with small deformation, ADINA's Timoshenko beam should be preferable, since the principal stretch $\lambda$ in this case will be very close to one so that the stretch-induced tension is small, and that the shear force may be a more important factor. This is because the shear force $=\partial M / \partial x$, where $M$ is the bending moment; hence, a shorter beam tends to produce a greater shear force.

On the other hand, it can be shown that a zero shear deformation will be approached as the wall thickness approaches zero [20]. Hence, it is expected that for a long and thin beam (as in our case), the shear term may not have significant effects, at least on steady solutions. 
It is not easy to compare the 2D-solid model used by ADINA with the FBM model directly, as the formula (11) used by ADINA is given in a general form only. However, it is clear that though both the models are suitable for large strain problems, different definitions of the stress and the strain are adopted by these models. In FBM the strain is defined as $\lambda-1$, where $\lambda$ is the principal stretch and the stress is the second Piola-Kirchhoff form. In the ADINA small strain model, the second Piola-Kirchhoff stress and Green-Lagrange strain are used, but in the ADINA large strain model, the strain is defined as $\ln \lambda$ (logarithmic strain) and the Cauchy stress is used. For problems with small strain, these definitions give similar results; however, for large strain problems, there will be differences. In other words, although in both FBM and ADINA models we assumed nonlinear geometry and linear material properties, the linearity of material is assumed between the defined stress and strain; therefore, the constitutive equations of the two large strain approaches (FBM and ADINA) for a hyper-elastic material will not be exactly the same. In practice, the precise form of the linearity of a hyper-elastic material should be determined by experiments, and one can then determine a suitable stress and strain. It is important to clarify this, if one needs to match the solution to an experiment that uses a specific material.

\section{RESULTS AND ANALYSIS}

\subsection{Parameters}

Three typical cases were selected for the investigation:

A. Self-excited oscillations from both FBM and ADINA are sustained.

B. Self-excited oscillation resulting from FBM is developed, while in ADINA it is damped out.

C. Oscillations resulting from both FBM and ADINA die away and the solutions approach the steady solution.

The corresponding dimensionless (dimensional) parameters are

Case A. $c_{\lambda}=139, R e=300, p_{\mathrm{e}}=4.73\left(E=0.01 \mathrm{MPa}, U_{0}=0.03 \mathrm{~m} / \mathrm{s}, p_{\mathrm{e}}=4.255 \mathrm{~Pa}\right)$,

Case B. $c_{\lambda}=250, R e=300, p_{\mathrm{e}}=1.95\left(E=0.018 \mathrm{MPa}, U_{0}=0.03 \mathrm{~m} / \mathrm{s}, p_{\mathrm{e}}=1.755 \mathrm{~Pa}\right)$,

Case C. $c_{\lambda}=2400, R e=500, p_{\mathrm{e}}=1.95\left(E=0.4785 \mathrm{MPa}, U_{0}=0.05 \mathrm{~m} / \mathrm{s}, p_{\mathrm{e}}=4.875 \mathrm{~Pa}\right)$.

For each of the cases tested, we consider the modelling assumptions listed in Table I, for the non-dimensional domain lengths

$$
L_{\mathrm{u}}=5, \quad L=5, \quad L_{\mathrm{d}}=30
$$

For simplicity, all results presented in the following are converted and shown in the non-dimensional variables as defined in Section 2.1.

\subsection{Steady solutions}

The steady elastic wall shape of the beam for the Cases A-C are shown in Figure 3 (left), for ADINA (2D-solid) large strain and ADINA (2D-solid) small strain, ADINA iso-beam, and FBM models. The corresponding pressure distributions along the elastic wall are also plotted in Figure 3 (right). In general, the four different models (FBM and ADINA) give similar steady results. For example in the Case C, $y(x=7.5)$ is 0.735796 for FBM, 0.734758 for large strain ADINA, and 0.736903 for small strain ADINA models. However, small differences exist in these different approaches. 
Table I. Modelling and numerical approaches used in the simulations.

\begin{tabular}{lllllll}
\hline Code & $\begin{array}{c}\text { Element } \\
\text { for beam }\end{array}$ & $\begin{array}{l}\text { Element } \\
\text { for fluid }\end{array}$ & $\begin{array}{c}\text { Structural } \\
\text { assumptions }\end{array}$ & $\begin{array}{l}\text { Time } \\
\text { integration }\end{array}$ & Fluid solver & Structure solver \\
\hline FBM & 3-node beam & $\begin{array}{l}\text { 6-node } \\
\text { triangle }\end{array}$ & $\begin{array}{l}\text { Large strain, large } \\
\text { displacement, without } \\
\text { shear strain, with } \\
\text { coupling terms }\end{array}$ & $\begin{array}{l}\text { First/second- } \\
\text { order, variable } \\
\text { time step }\end{array}$ & $\begin{array}{l}\text { Second } \\
\text { order, } \\
\text { adaptive } \\
\text { mesh }\end{array}$ & Second order \\
ADINA & $\begin{array}{l}\text { 9-node 2D } \\
\text { solid }\end{array}$ & $\begin{array}{l}\text { 9-node } \\
\text { quadrilateral }\end{array}$ & $\begin{array}{l}\text { Large/small strain, } \\
\text { large displacement, } \\
\text { with shear strain }\end{array}$ & $\begin{array}{l}\text { First/second- } \\
\text { order, fixed } \\
\text { time step }\end{array}$ & $\begin{array}{l}\text { First- } \\
\text { order } \\
\text { upwind }\end{array}$ & Second order \\
ADINA & $\begin{array}{l}\text { 4-node } \\
\text { iso-beam }\end{array}$ & $\begin{array}{l}\text { 9-node } \\
\text { quadrilateral }\end{array}$ & $\begin{array}{l}\text { Small strain, large } \\
\text { deformation, with } \\
\text { shear strain, without } \\
\text { coupling terms }\end{array}$ & $\begin{array}{l}\text { First/second- } \\
\text { order, fixed } \\
\text { time step }\end{array}$ & $\begin{array}{l}\text { First- } \\
\text { order } \\
\text { upwind }\end{array}$ & Second order \\
& & & & & & \\
\hline
\end{tabular}

Note: For all cases, the effect of wall inertia is not included.

The two small strain ADINA models, 2D solid and iso-beam, give almost identical results and predict the minimum deformation of the wall, where the large strain ADINA model predicts the maximum deformation. We note that although both FBM and ADINA 2D solid assumed large strain in their modelling, the deformation predicted from ADINA 2D solid seems to be greater than that from FBM. This is presumably because there is a stretch-induced tension force in FBM, which tends to resist and reduce the stretching deformation.

The streamlines of the steady flow obtained using the different models for Case A are shown in Figure 4. There are no significant differences in these models, at least for the steady flow case. This is to be expected, as all these models employ the same fluid modelling (Navier-Stokes equations); hence, any difference in the model can only come from the solid modelling. The same results are obtained for Cases B and C (not shown).

\subsection{Unsteady solutions}

Time-dependent simulations are performed. For the FBM model, a variable time step control is employed with a total error tolerance of $10^{-6}$ [17], for ADINA models, the fixed time step of 0.087 is used after an essential test of time steps (i.e. for time step of $0.11,0.087$, and 0.072 , the difference in the results is within $1 \%$ in the maximum amplitude of the wall shape, with no observable change in the oscillatory waveforms). The time history of the $y$-displacement of the material point initially at the mid-point of the elastic beam $(x=7.5)$ is shown in Figures $5-7$ for Cases A, B, and C, respectively.

It is interesting to see that for all three cases, the iso-beam and 2D-solid small strain models in ADINA give more or less identical results, both in steady and unsteady simulations. It is not clear to us if the ADINA 2D-solid element configurations have included the coupling terms as in the FBM; however, the fact that all results using the small strain 2D-solid elements agree with the iso-beam model (see below) strongly suggests that these terms are absent in ADINA 2D-solid models, i.e. it basically represents a Timoshenko beam without longitudinal stretch, at least for the small strain case. This lack of coupling between the axial and normal deformations in ADINA models could be a major reason for the differences observed in the unsteady simulations. 

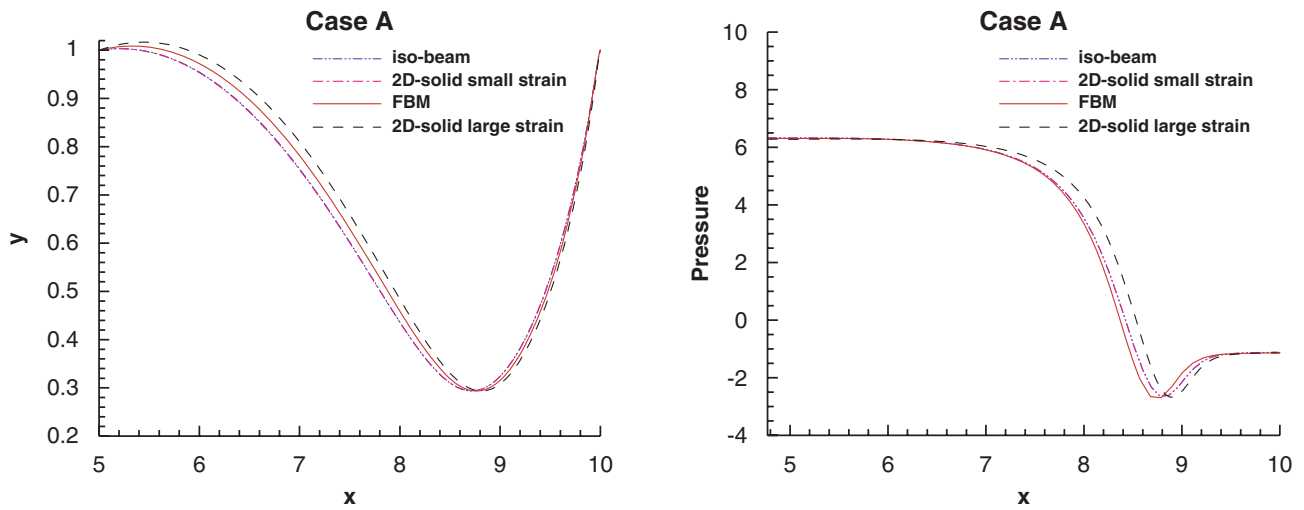

Case B
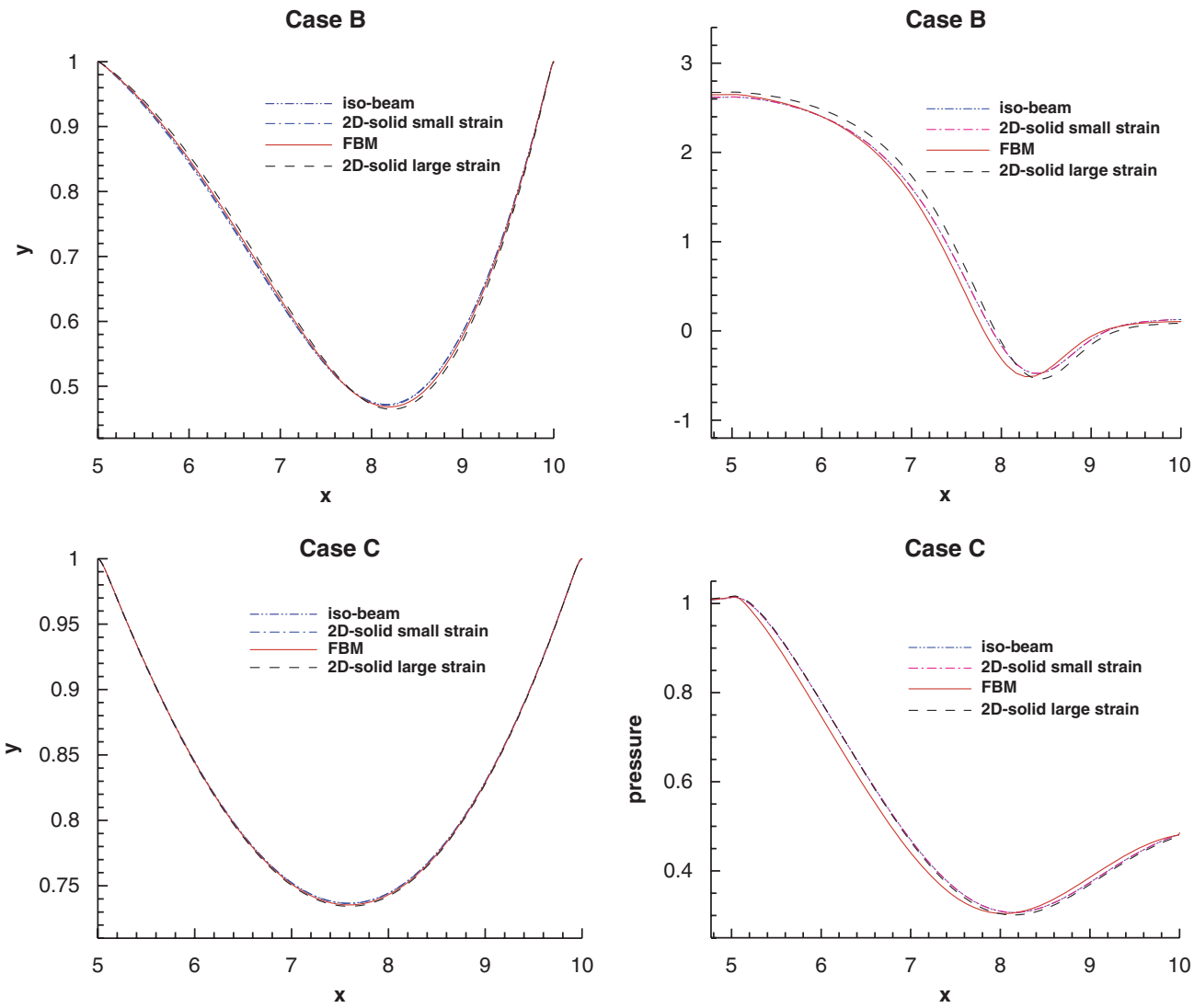

Figure 3. Comparison of steady solutions from ADINA 2D-solid large strain (dashed curve), ADINA 2D-solid small strain (dash-dotted curve), ADINA iso-beam (dash-dot-dot) and FBM (solid curve) models. On the left is the wall shape of the elastic beam, and on the right, is the pressure distribution along the elastic wall. Note the two ADINA models (2D-solid small strain and iso-beam) give almost identical results. 

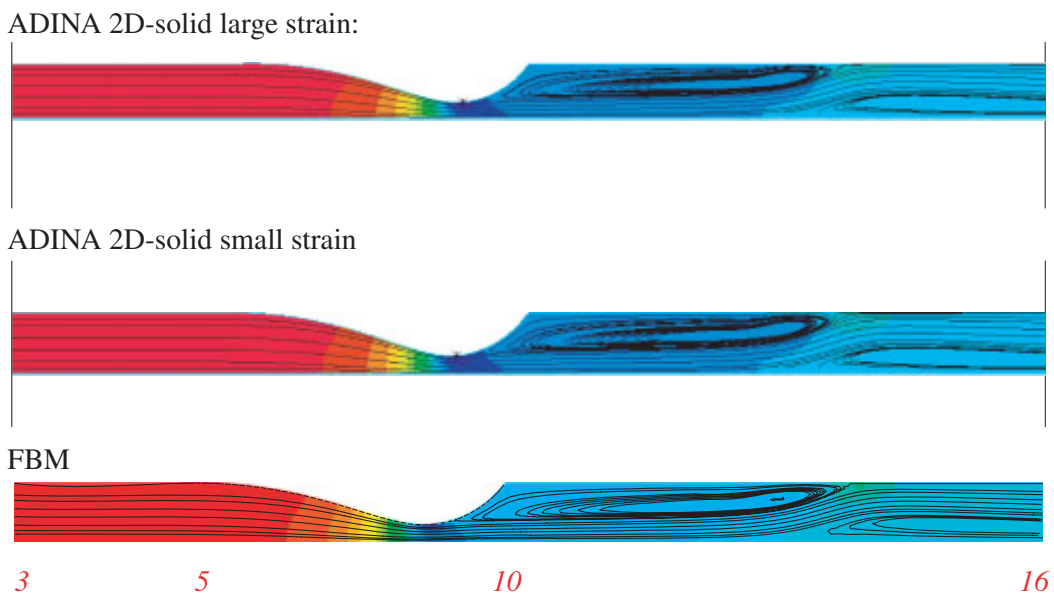

Figure 4. The steady streamline functions for Case A, obtained using the ADINA 2D-solid large strain, small strain, and FBM models. ADINA iso-beam model gives almost identical results as 2D-solid small strain (not shown). Only part of the domain is shown.

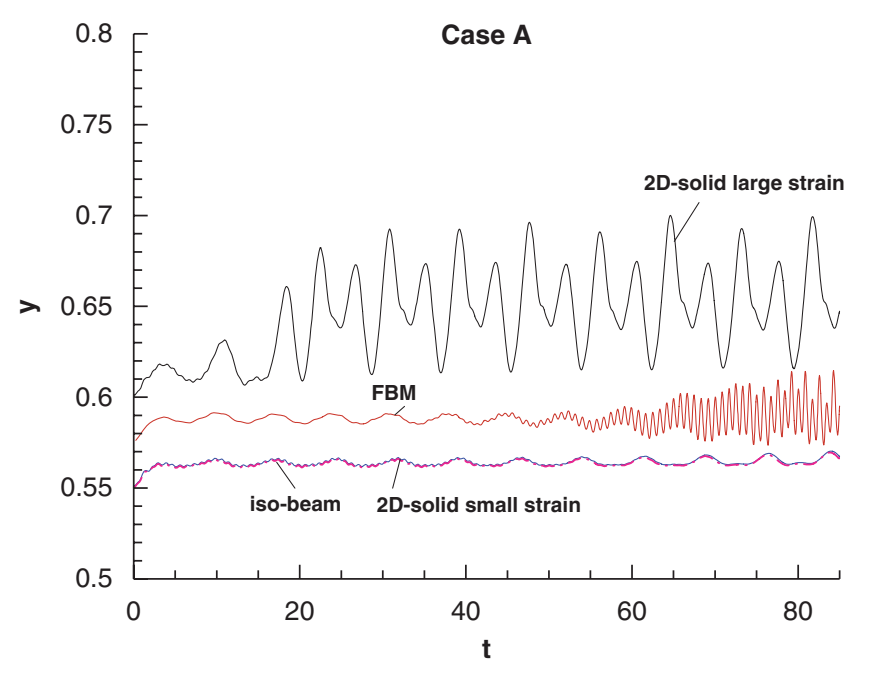

Figure 5. Comparison of unsteady vibrations for Case A. The history of the wall displacement $y(t)$ at $x(t=0)=7.5$ is plotted for ADINA 2D-solid large strain, ADINA 2D-solid small strain (dash-dot), ADINA iso-beam, and FBM models.

For Case A (Figure 5), it is noted that the initial vibrations of all four models are similar in terms of the oscillating frequency and pattern (for $t<10$ ). However, the discrepancy soon shows up as time progresses. For ADINA 2D-solid large strain, a period doubling is developed with relatively greater amplitude, and the beam is oscillating at more or less neutrally stable mode (for the time period shown). For ADINA 2D-solid small strain and iso-beam, there is a small amplitude, (10 times) higher-frequency oscillation superimposed on the original regular oscillation, which, 


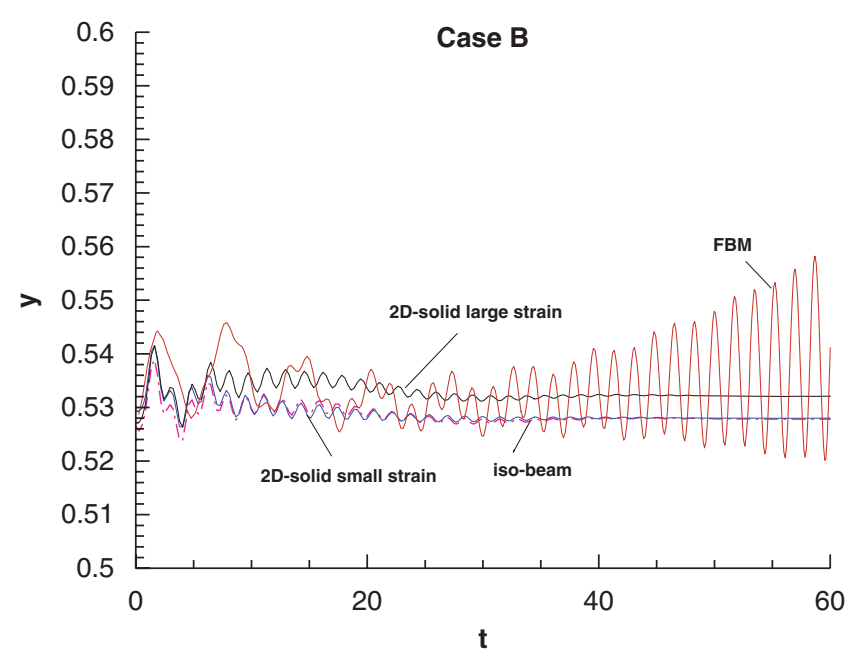

Figure 6. Same as Figure 5, but for Case B.

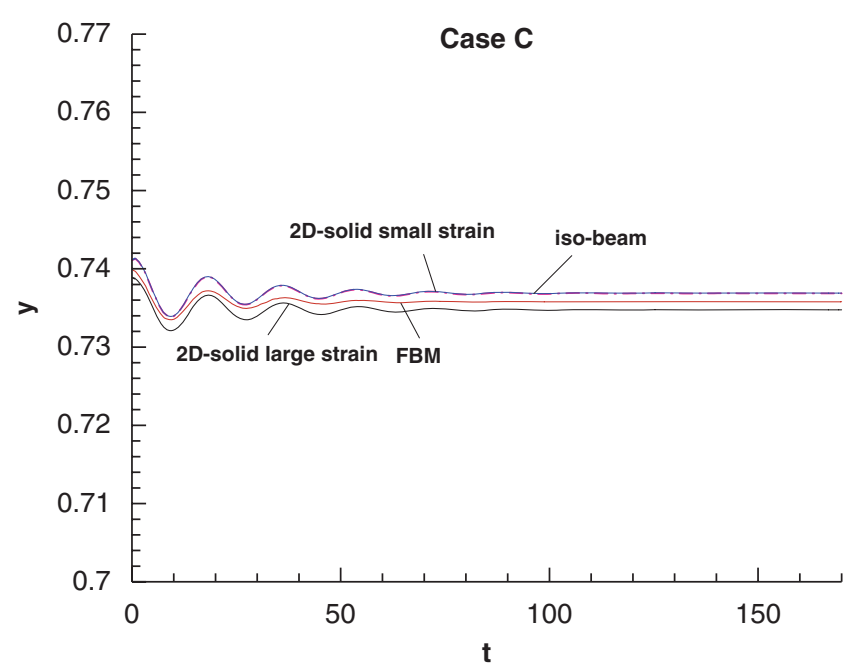

Figure 7. Same as Figure 5, but for Case C. Although all models approach their corresponding steady solutions, there exists a small discrepancy in these steady solutions due to the modelling assumptions.

however, is damped out after $t>60$. The low-frequency oscillation is sustained at a neutral state. The FBM model exhibits a very similar trend as ADINA small strain models until $t \sim 45$, except that in FBM the secondary oscillation is not damped out, but becomes dominant after $t=45$. This causes the system to become unstable.

The comparison of the three models in Case B is shown in Figure 6. In this case, all three ADINA models give very similar results, with almost the same frequency and oscillating pattern, and the oscillation dies out as $t>40$. The FBM model, on the contrary, behaves very differently. 
The oscillation starts with a much lower frequency, but it is soon taken over by an oscillation with a higher frequency and the system becomes unstable. Finally, for Case C (Figure 7), all four models predict more or less the same result: the transient perturbations die out in time and the system approaches the corresponding steady solution. It is noted that the differences among these models in Case $\mathrm{C}$ are not as great as those in Cases A and B. The reason is that in Cases A and $\mathrm{B}$, the system is very close to being either neutrally stable or unstable, which means that a small discrepancy may lead to large deviation in the unsteady behaviour, while for Case $\mathrm{C}$, the system is in the stable region and the steady solution becomes dominant. As we showed in Section 3.1, the difference in the model assumptions does not have much impact on the steady solutions.

\subsection{Linear stability analysis and the transient modes}

To fully understand the transient behaviour of the three cases, in the following the linear stability analysis to the steady solutions is carried out using the FBM eigenvalue solver. As ADINA 8.4 does not have an eigen-solver for this problem, comparisons will be made with the ADINA results using the Fourier transform.

The linear stability computation shows that for Case A, both mode- 2 and mode- 4 are unstable modes. The growth rate for mode- 2 is slightly greater than that of mode- 4 , but the frequency is almost ten times smaller. On the other hand, for Case B, mode- 3 is the unstable one. In both cases, the frequency of mode- 3 is almost four times greater than mode-2, which is consistent with the earlier studies for this system [5,17]. Note that, in general, there can be more than one mode of mode-1 or other; however, only the ones with the greatest growth rates are listed in Table II. Other modes have much faster decay rates and cannot persist for any length of time in the transient results. Indeed, for Case $\mathrm{C}$, although all modes are stable ones, the ones with the greatest growth rates are mode- 2 , and two mode- 1 s, shown as mode- $1_{1}$ and mode- $1_{2}$ in Figure 8 . The difference

Table II. The eigenvalues of the modes shown in Figure 8 for Cases A, B, and C.

\begin{tabular}{lcc}
\hline & $\omega_{r}$ & $\omega_{i}$ \\
\hline Case $A$ & & \\
Mode-2 & $\mathbf{0 . 1 6 3 1}$ & $\mathbf{0 . 7 1 9 6}$ \\
Mode-3 & -0.03155 & 3.6355 \\
Mode-4 & $\mathbf{0 . 1 3 3 0 5}$ & $\mathbf{7 . 3 7 2 1}$ \\
Case B & & \\
Mode-1 & -0.1662 & 0.1586 \\
Mode-2 & -0.05812 & 1.0021 \\
Mode-3 & $\mathbf{0 . 0 5 2 1 2}$ & $\mathbf{3 . 7 1 4 6}$ \\
Mode-4 & -0.2282 & 7.3839 \\
Case C & & \\
Mode-1 & & 0.3452 \\
Mode- $1_{2}$ & -0.05585 & 0.02891 \\
Mode-2 & -0.1870 & 2.5809 \\
\hline
\end{tabular}

Note: The mode number $i, i=1,2,3, \ldots$, here means that the perturbation to system contains $i$ half-wavelengths along the length of the beam. The ones in bold indicate the unstable modes. Note only the fastest growing modes for each case are listed here. 

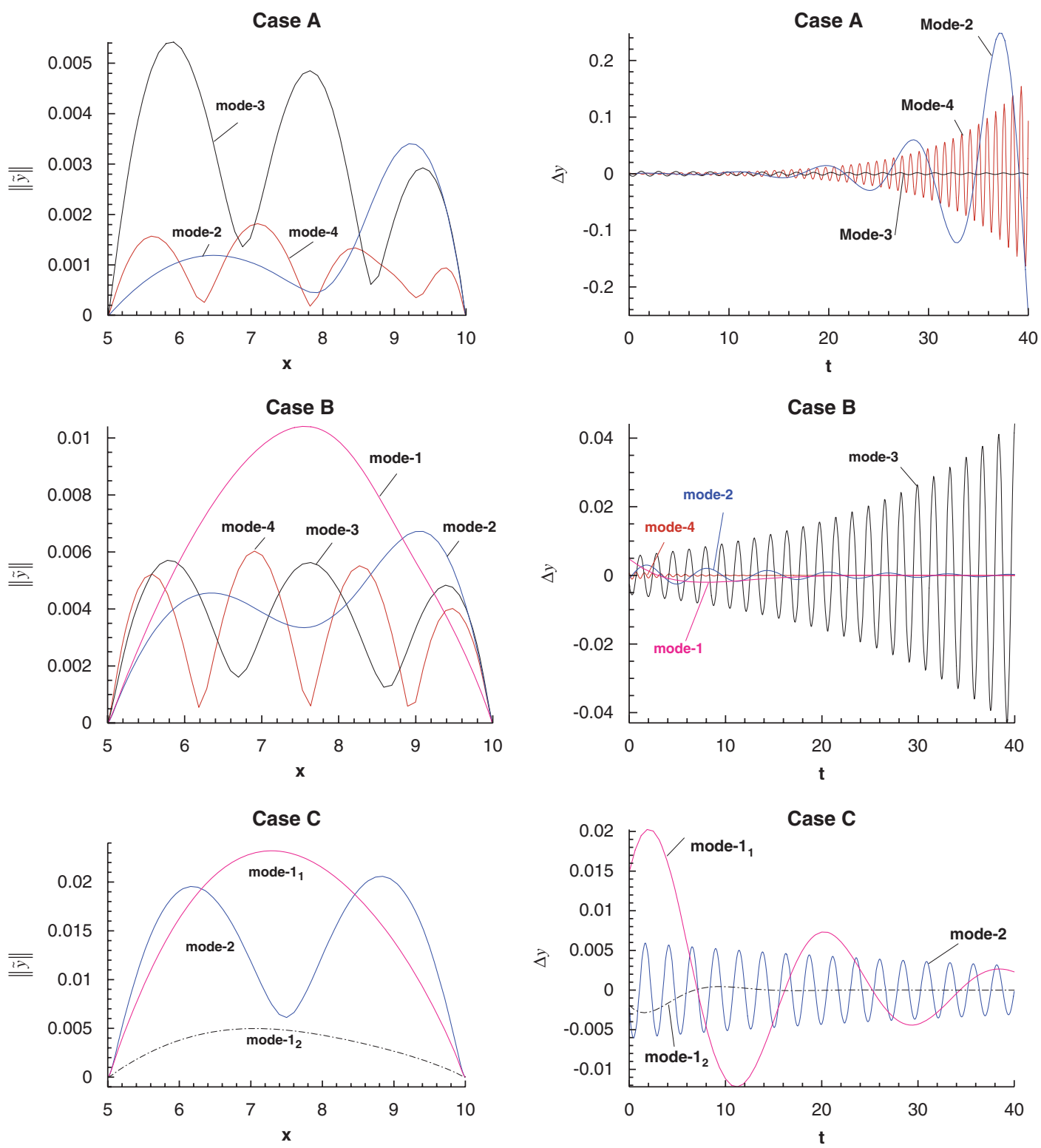

Figure 8. Eigenmodes of Cases A-C. (left) The oscillating amplitude in $y$-direction along the beam and (right) the time history of the $y$ component of a point on the beam initially at $x=7.5$.

between these two modes is not only in their decay rates (mode- $1_{2}$ decays much faster), but also in the manner in which they oscillate. The oscillating two mode- 1 beam shapes at six time instants in a cycle are shown in Figure 9. Figure 9 shows that in mode- $1_{1}$, the beam is oscillating more around the corresponding steady solution with the first half of the beam more expanded and second 

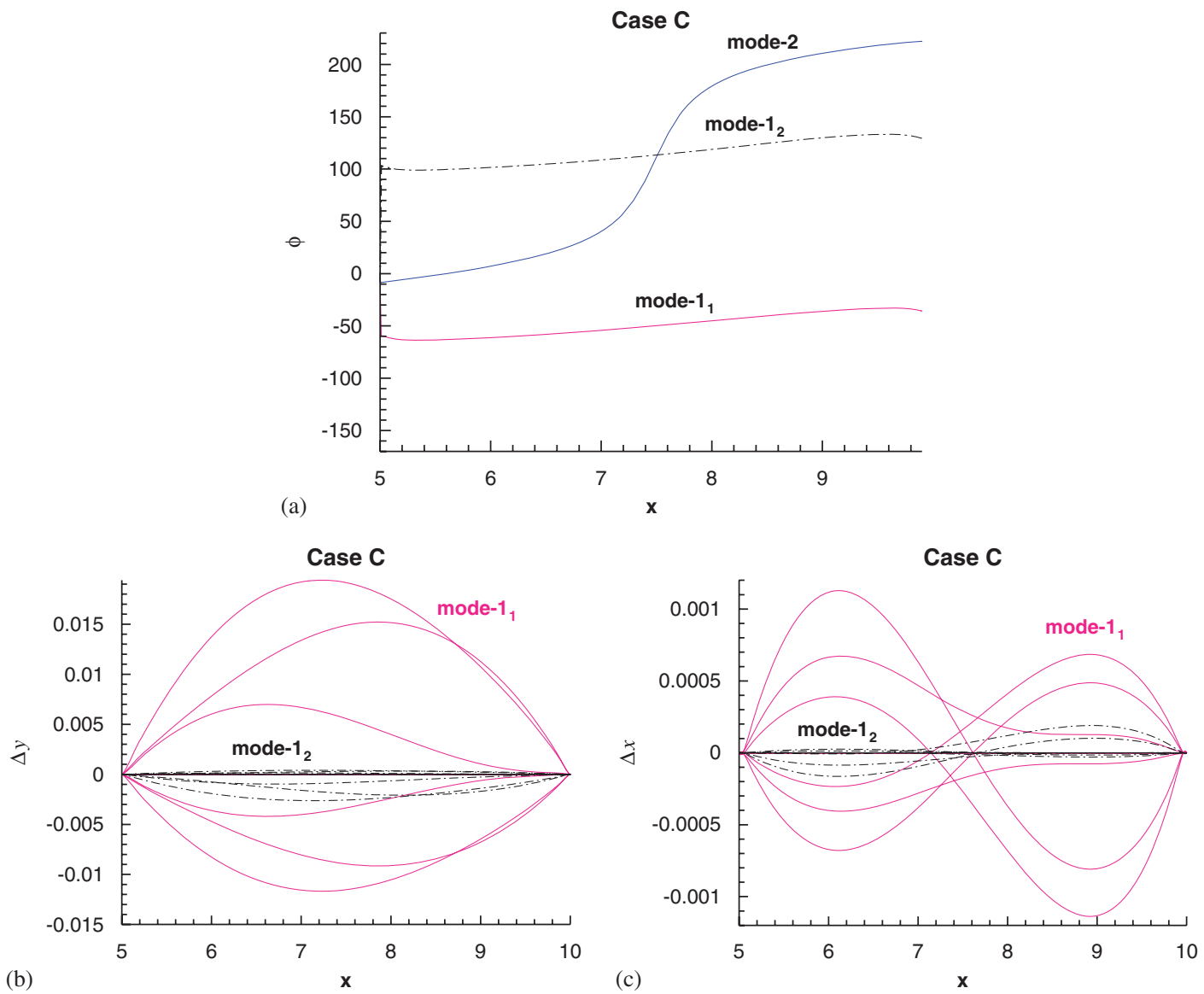

Figure 9. (a) The phase plot for different modes in Case $\mathrm{C}$ and the two mode- 1 beam shapes at six time equal spaced instants in a cycle in terms of (b) $x$-component; and (c) $y$-component. Mode-1 1 (solid), Mode-12 (dash-dot).

half more compressed in the $x$-direction, while in mode- $1_{2}$ the beam is oscillating more inwards, and the first half of the beam is more compressed and the second half of beam is more expanded in the $x$-direction.

By performing a Fourier transform analysis for the full unsteady solutions for both FBM and ADINA, we can show that these oscillations consist of principal oscillating modes with the frequencies shown in Table III.

Table III shows that for Case A, the most unstable oscillation mode- 2 is present in all the unsteady simulations. For Case B, both the unstable mode- 3 and the stable mode- 2 are present in all the simulations. In Case $\mathrm{C}$, mode- $1_{1}$ is the common feature in all the unsteady simulations. The overall agreement with the eigen-solver is good, though there are some notable differences in the frequencies predicted, which could be due to both the solver and the modelling differences. We also note that these unsteady oscillations have gone through a fully non-linear process, which 
Table III. Frequencies estimated using the Fourier transform for the full unsteady simulations using ADINA and FBM.

\begin{tabular}{|c|c|c|c|}
\hline & Case A & Case B & Case $\mathrm{C}$ \\
\hline FBM & $\begin{array}{l}0.88 \text { (mode- }) \\
3.66 \text { (mode- } 3) \\
7.49 \text { (mode- } 4)\end{array}$ & $\begin{array}{c}1 \text { (mode- } 2) \\
3.64(\text { mode- } 3)\end{array}$ & $0.33\left(\right.$ mode- $\left.\mathrm{I}_{1}\right)$ \\
\hline ADINA small strain 2D solid & $\begin{array}{c}0.88 \text { (mode-2) } \\
1.76 \\
7.91 \text { (mode- } 4)\end{array}$ & $\begin{array}{l}1.17 \text { (mode-2) } \\
4.17 \text { (mode-3) }\end{array}$ & $0.37\left(\right.$ mode $\left.-\mathrm{I}_{1}\right)$ \\
\hline ADINA large strain 2D solid & $\begin{array}{c}0.63 \\
0.88 \text { (mode- } 2) \\
1.56\end{array}$ & $\begin{array}{c}1.1(\text { mode- } 2) \\
3.87(\text { mode- } 3)\end{array}$ & $0.37\left(\right.$ mode- $\left._{1}\right)$ \\
\hline ADINA small strain iso-beam & $\begin{array}{c}0.88 \text { (mode-2) } \\
1.76 \\
7.91 \text { (mode- } 4)\end{array}$ & $\begin{array}{l}1.17 \text { (mode-2) } \\
4.17 \text { (mode-3) }\end{array}$ & $0.37\left(\right.$ mode- $\left.\mathrm{I}_{1}\right)$ \\
\hline Eigen-modes from the FBM eigen-solver & $\begin{array}{l}0.7196 \text { (mode- } 2) \\
3.635 \text { (mode- } 3) \\
7.372 \text { (mode- } 4)\end{array}$ & $\begin{array}{l}0.1586 \text { (mode-1) } \\
1.0021 \text { (mode- }) \\
3.7146 \text { (mode-3) } \\
7.3839 \text { (mode-4) }\end{array}$ & $\begin{array}{c}0.3452\left(\text { mode }-1_{1}\right) \\
0.0289\left(\text { mode- } 1_{2}\right) \\
2.5809(\text { mode }-2)\end{array}$ \\
\hline
\end{tabular}

Note: The mode number from the full simulations is speculated by a comparison with the prediction of the corresponding eigen-solver, which is listed in the bottom row.

may have shifted away slightly from the linear stability behaviour at the onset, as predicted by the FBM eigen-solver.

\subsection{Influence of the shear strain}

In this section, we examine the sensitivities of the unsteady solutions to the model assumptions, especially concerning the shear strain. This is important since the shear strain contribution to shear stress is neglected in the FBM model (though the shear stress is still present), and we need to know if this approximation contributes to the discrepancies in unsteady behaviour for the three cases investigated. For simplicity, the ADINA large strain 2D-solid model is used for Case A only, to check how important the shear strain is.

The shear strain distribution for Case A steady flow is shown in Figure 10 (dashed). As there is no option to switch off the shear term in ADINA, here we will try to reduce the magnitude of shear strain at the two ends of the beam by lifting the fixity of the $z$-rotational degree of freedom at the two ends of the beam (i.e. pinned ends). The result is shown in Figure 10 (solid curve). It is clear that although the magnitude of the shear strain is reduced at the downstream end for the pinned ends, the overall shear strain distribution is not negligibly small along the beam.

We now compare the steady and the unsteady wall shapes with clamped and pinned ends in Figure 11. It is interesting to see that there are no significant differences in the steady solutions, but the unsteady simulations show some discrepancies, although the general trend is similar. This demonstrated again how sensitive the unsteady simulations are to modelling assumptions. In other words, the shear strain term neglected in the FBM model could contribute to the differences in the unsteady oscillations produced by the FBM and ADINA large strain models. 


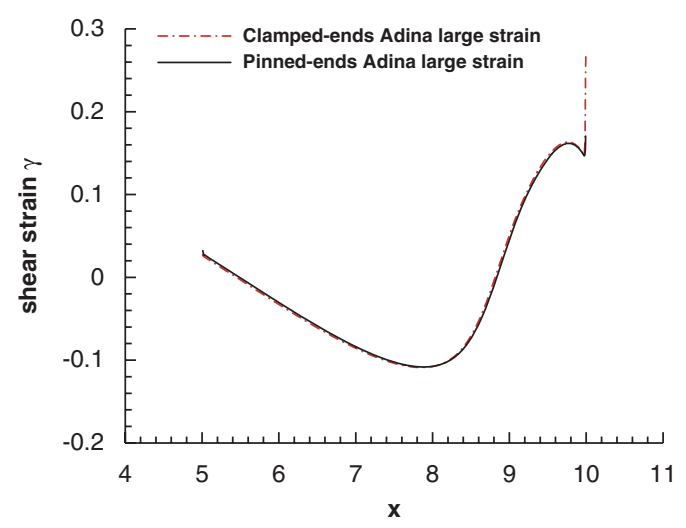

Figure 10. Distribution of shear strain $\gamma$ for Case A along the beam using the ADINA large strain 2D-solid model with clamped ends (dashed) and pinned ends (solid). The maximum shear strain occurs at the downstream end of the beam, and is reduced by allowing the ends to rotate.
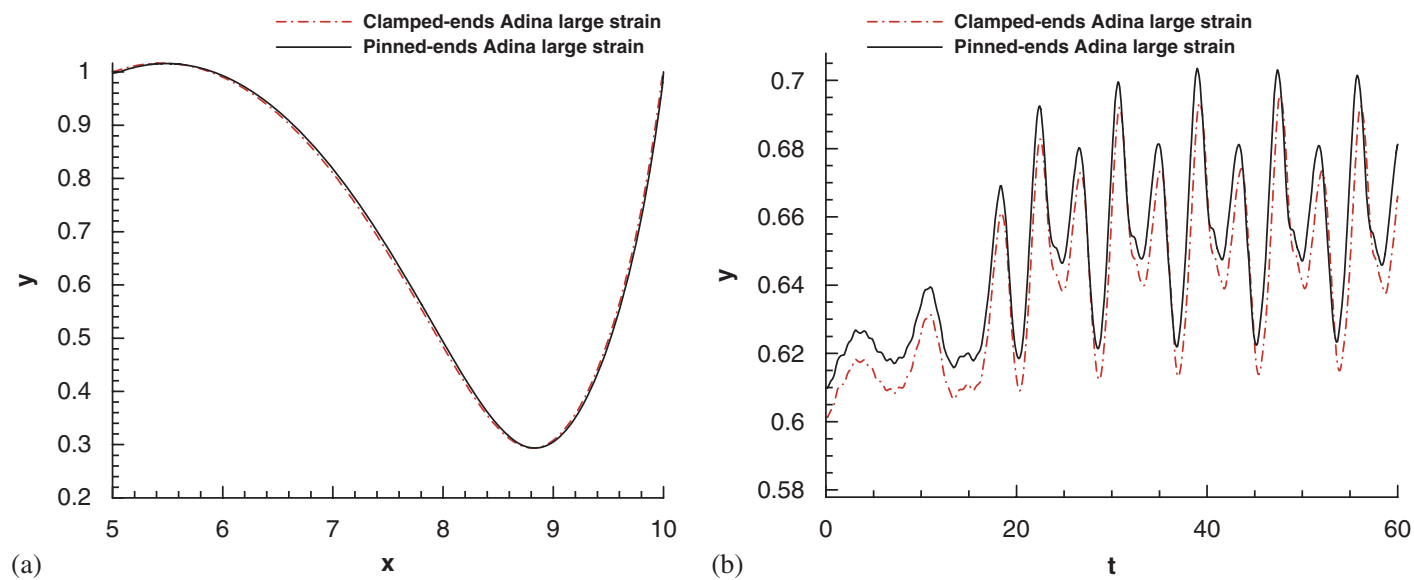

Figure 11. (a) The steady wall shape and (b) the time history at $x=7.5$ initially for Case A, with and without the rotation of the two ends released.

\subsection{Influence of the time integration scheme}

The influence of the numerical diffusion on the unsteady behaviour of the system is examined by using first- and second-order time integrations in ADINA 2D-solid large strain and FBM models, and again, only results for Case A are shown. For the purpose of comparison, the same time step of 0.087 is used for both the first- and second-order time integrations.

Figure 12 shows that the first-order time integration is numerically too diffusive and tends to damp out the higher-order self-excited oscillations. This difference between the first- and secondorder time integration becomes smaller as the time step size in the first-order time integration is significantly reduced (at a cost of much greater computational time). A similar trend is observed in the other two cases. In addition to the order of time integration, it is noted that the order of 


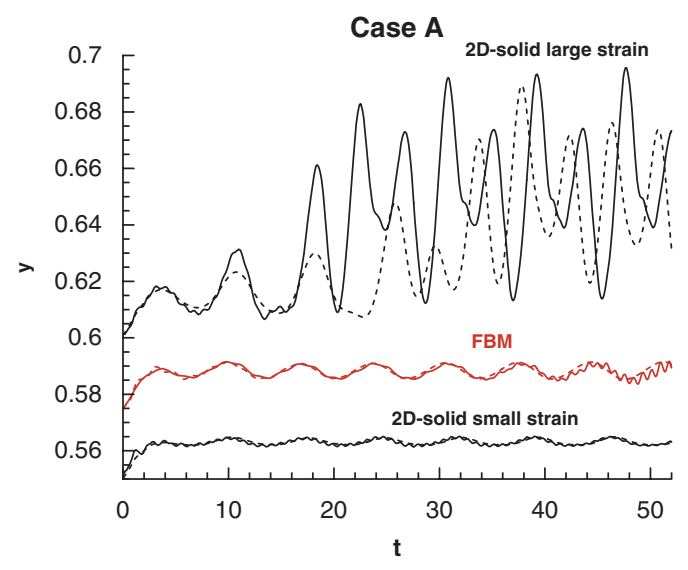

Figure 12. The beam deformation history $y(t)$ for Case A at $x(t=0)=7.5$, with first- (dashed) and second-order (solid) time integrations in ADINA and FBM.

fluid solver may have some impact, especially on the transient behaviour. The fluid solver used in FBM is second order [6], but in ADINA's models, a first-order upwind scheme is used for the fluid elements. ${ }^{\S}$ This presumably would make the ADINA models slightly more numerically diffusive, which may also be a contributing factor to the differences observed in the models (although every effort is made to reduce this effect by increasing the number of grid points).

\subsection{Sensitivity of the system stability to boundary conditions}

In this section, we test the sensitivity of the system neutral stability to boundary conditions using the eigen-solver of FBM. In all the studies carried out so far, only one type of boundary conditions is considered: the inlet flow rate is specified with a velocity parabolic profile. However, we could equally consider the case where the driving pressure is specified at the channel inlet. In both cases, the downstream end is set to be stress free. The motivation comes from the observation that Jensen and Heil [10] predicted only mode-1 instability for the system, whereas Luo et al. [17] observed higher modes (modes 2, 3, 4). The major modelling differences lie in the inlet boundary conditions. In [10], the oscillations were reproduced numerically only when the upstream pressure was fixed, not the upstream flow rate as here. This is because their analytical model predicts instability only when the downstream rigid segment is longer than the upstream one; thus, prescribing the upstream flow rate (equivalent to a very large upstream length) is the stablest possible state for this mode [10].

To see if the inlet boundary condition can change the system stability and its oscillatory modes, we impose the two boundary conditions for a testing point: $c_{\lambda}=1760, p_{\mathrm{e}}=1.95, \operatorname{Re}=300$. This is a point that is located almost at the mode- 2 neutral curve with flow prescribed at the inlet [17].

\footnotetext{
$\S$ ADINA 8.4 also has FCBI-C element that is capable of a second-order upwind accuracy; however, this element does not converge well for the FSI problem studied here.
} 

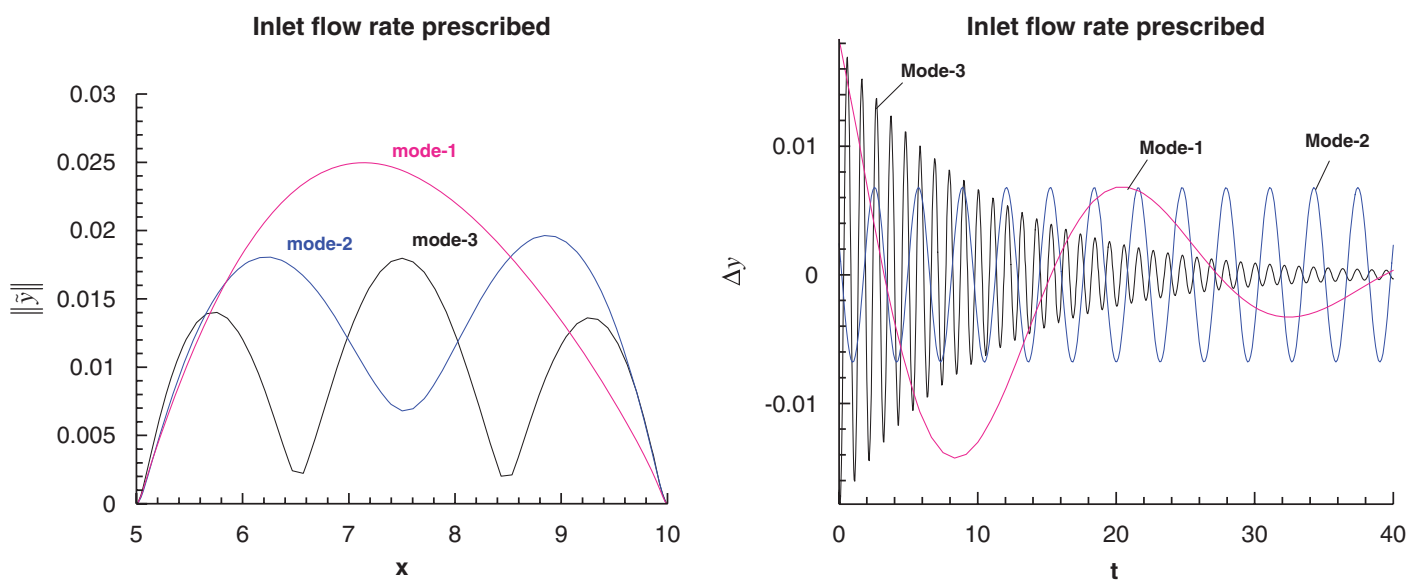

Figure 13. The eigen modes of the testing point with inlet flow prescribed. (left) The amplitude and (right) the time histories of the $y$-component of a point initially at $x=7.5$.
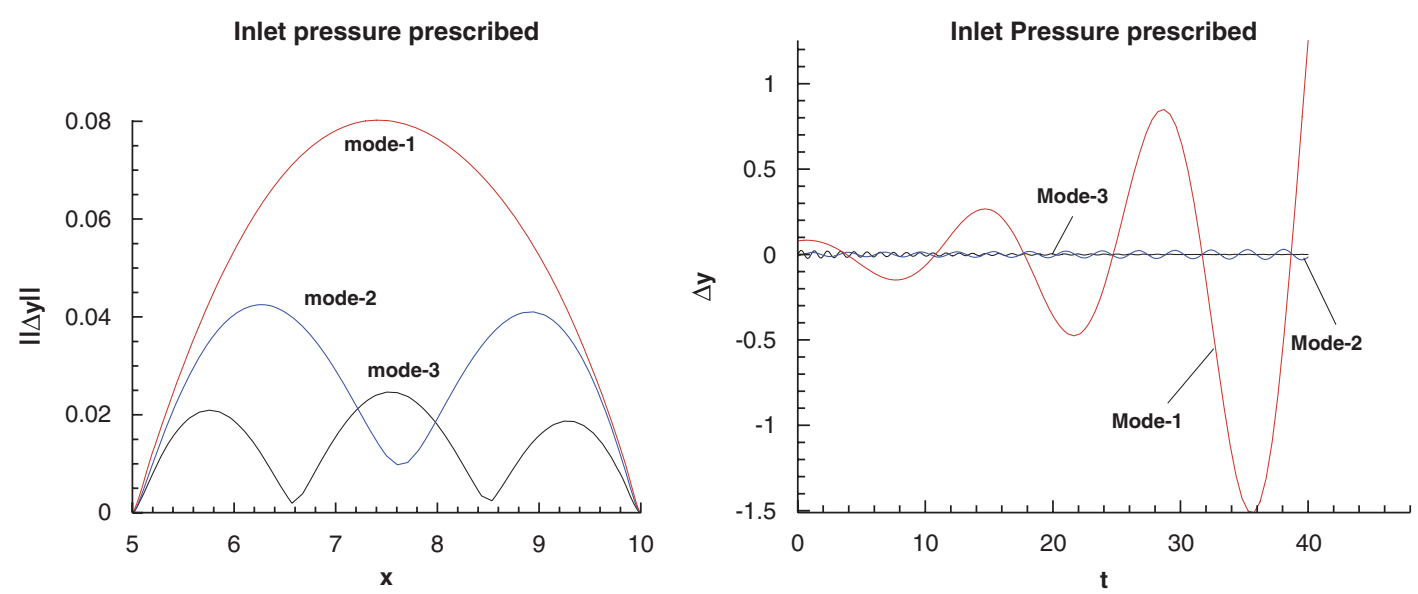

Figure 14. Same as Figure 13, but with the inlet pressure prescribed.

The eigenmodes computed for the two boundary conditions are plotted in Figures 13 and 14, respectively. The corresponding eigenvalues are shown in Table IV. It is interesting to see that when the inlet flow rate is prescribed, the system undergoes a mode- 2 neutral oscillation, with all the other modes decaying. However, for exactly the same parameters but with the inlet pressure prescribed, in addition to the unstable mode- 2 oscillation (with a slightly higher frequency), there exist two additional unstable instabilities: one is the mode- 1 instability, which has much higher growth rate than that of mode- 2 and the other is the divergence, a static instability with a zero frequency. We have not found, in the range of numerical simulations carried out, the divergence stability for a prescribed flow rate boundary condition case. 
Table IV. The eigenvalues obtained for the two different inlet boundary conditions.

\begin{tabular}{lcc}
\hline & $\omega_{r}$ & $\omega_{i}$ \\
\hline Inlet flow prescribed & & \\
Mode-3 & $-9.92 \mathrm{E}-002$ & 5.97 \\
Mode-2 & $-7.32 \mathrm{E}-005 \approx 0$ (neutral) & 1.98 \\
Mode-1 & $-6.08 \mathrm{E}-002$ & 0.261 \\
Inlet pressure prescribed & & \\
Mode-3 & $-9.42 \mathrm{E}-002$ & 6.089 \\
Mode-2 & $\mathbf{1 . 6 2 E}-\mathbf{0 0 2}$ & 2.192 \\
Mode-1 & $\mathbf{5 . 9 9 E}-\mathbf{0 0 2}$ & 0.575 \\
Divergence & $-3.78 \mathrm{E}-002$ & 0.0 \\
\hline
\end{tabular}
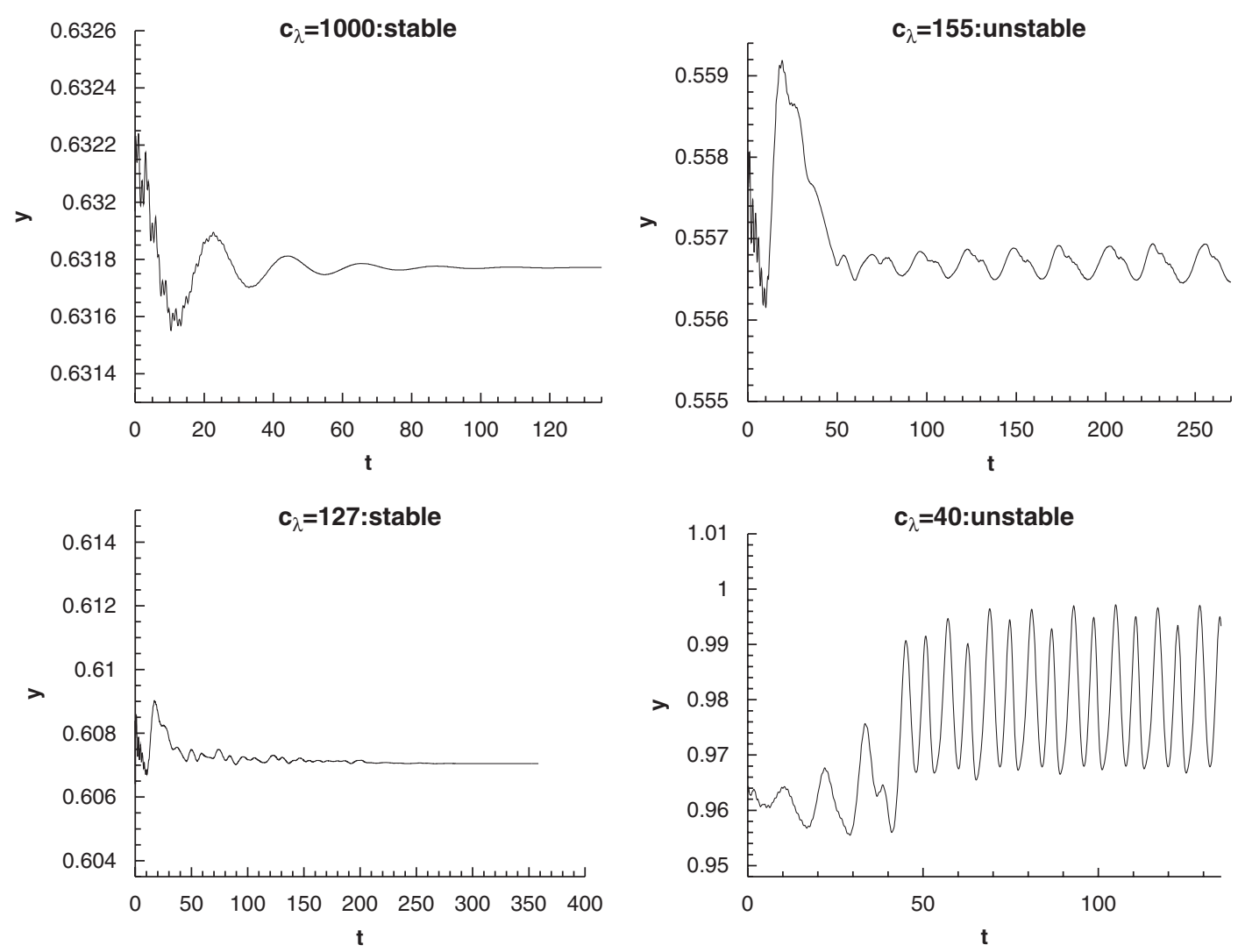

Figure 15. Evidence of a cascade structure using the ADINA 2D-solid large strain model: as the wall stiffness $c_{\lambda}$ is reduced, the system passes through a succession of unstable zones, separated by the stable zones. In all simulations we have $R e=450, P e=1.95$, with the inlet flow rate prescribed. 


\subsection{Evidence of the cascade structure}

Luo et al. [17] discovered a cascade stability structure using the FBM model, which shows that the neutral stability of this system appears in a cascade consisting of different disturbance modes. This cascade structure shows that in the $R e-c_{\lambda}$ space, depending on the inlet boundary condition and the Reynolds number, the system may first lose stability to mode- 2 perturbations as wall stiffness is lowered to a critical value. As the wall stiffness is further reduced, the system is re-stabilized before going through a sequence of higher-mode unstable zones. Sometimes a stable 'tongue' is formed as a result of a gap between mode- 2 and mode- 3 neutral branches.

In this section, we test if such a cascade structure exists using different modelling assumptions. This is done by using the ADINA 2D-solid large strain model. As the current version of ADINA does not have an eigen-solver, it is not possible to locate the neutral curves as was done by Luo et al. [17]. The best we could do is to run the time-dependent simulations (albeit with a small initial numerical perturbation) around the steady solutions. If the initial perturbation dies out as time increases, then such a solution is deemed to be stable; otherwise, it is unstable. Using this rather crude approach, we cannot obtain the neutral points accurately. However, if a cascade structure does exist, then as the wall stiffness $c_{\lambda}$ for a fixed $R e$ and $P e$ (say, $R e=450, P e=1.95$ ) is reduced, the system should go through a sequence from stable to unstable, to stable and unstable again. This is indeed the case, as shown in Figure 15.

Figure 15 shows that with the ADINA 2D-solid large strain model, as the wall stiffness $\mathrm{c}_{\lambda}$ is reduced, the system passes through a succession of unstable zones, separated by the stable zones. This represents a typical cascade type of system stability [17]. Owing to the sensitivities of the modelling assumptions shown in the previous sections, quantitative agreement between the unstable zones predicted by ADINA and the FBM model is not to be expected; however, the similar pattern of the system behaviour is clearly demonstrated. This is important, and highlights the robustness of the cascade structure predicted by Luo et al. [17].

\section{CONCLUSIONS}

This paper studies the effects of the modelling assumptions on the unsteady behaviour of collapsible channel flows. Using the in-house software, FBM, as well as the commercial package ADINA 8.4 , we are able to investigate the sensitivity of the self-excited oscillations to various modelling assumptions, such as the strain definition, large and small strain assumptions, Timoshenko beam, Euler beam, shear strain effects, coupling between axial and normal stretch, boundary conditions, and accuracy of the numerical schemes. While the steady solutions seem to be quite robust to all these different modelling approaches, the unsteady solutions, especially the unstable solutions, can be highly sensitive and have different responses. Among these assumptions, this study shows that allowing the elastic wall to undergo a large strain deformation and including the coupling terms may have a significant impact. In addition, the shear strain term present in ADINA models and a different definition for the large strain problem adopted by the two approaches could all contribute to the different responses of the unsteady solutions. In addition, a second-order numerical scheme in both time and space would reduce the numerical diffusion significantly, and therefore may more accurately predict the onset of the self-excited oscillations, although using a finer grid and smaller time step can reduce this impact. Finally, the inlet boundary condition also has a strong influence on the system behaviour. If the flow rate at the channel entrance is specified, the system tends to 
be more stable than if the pressure is specified. Moreover, the system imposing the latter condition is much more vulnerable to mode- 1 perturbations and can also lose instability through divergence. This is consistent with the previous studies of the same system $[10,17]$. Finally, we note that despite all the modelling differences, the previously identified cascade stability structure is shown to exist when a different numerical model is used.

\section{ACKNOWLEDGEMENTS}

This work is supported by the U.K. Royal Society of London, the U.K. Royal Academy of Engineering, and the Tianjin Municipal Science and Technology Commission, People's Republic of China.

\section{REFERENCES}

1. Bertram CD. Two modes of instability in a thick-walled collapsible tube conveying a flow. Journal of Biomechanics 1982; 15:223-224.

2. Bertram CD, Raymond CJ, Pedley TJ. Mapping of instabilities for flow through collapsed tubes of differing length. Journal of Fluids and Structures 1990; 4:125-153.

3. Bertram CD, Elliot NSJ. Flow-rate limitation in a uniform thin-walled collapsible tube, with comparison to a uniform thick-walled tube and a tube of tapering thickness. Journal of Fluids and Structures 2003; 17:541-559.

4. Shapiro AH. Steady flow in collapsible tubes. Transactions of the ASME, Journal of Biomechanical Engineering 1998; 99:126-147.

5. Jensen OE. Instabilities of flow in a collapsed tube. Journal of Fluid Mechanics 1990; 220:623-659.

6. Luo XY, Pedley TJ. A numerical simulation of unsteady flow in a two-dimensional collapsible channel. Journal of Fluid Mechanics 1996; 314:123-144.

7. Luo XY, Pedley TJ. The effects of wall inertia on flow in a 2-D collapsible channel. Journal of Fluid Mechanics 1998; 363:253-280.

8. Luo XY, Pedley TJ. Flow limitation and multiple solutions in 2D collapsible channel flow. Journal of Fluid Mechanics 2000; 420:301-324.

9. Pedley TJ, Luo XY. Modelling flow and oscillations in collapsible tubes. Theoretical Computational Fluid Dynamics 1998; 10:277-294.

10. Jensen OE, Heil MH. High-frequency self-excited oscillations in a collapsible-channel flow. Journal of Fluid Mechanics 2003; 481:235-268.

11. Cai ZX, Luo XY. A fluid-beam model for flow in collapsible channels. Journal of Fluids and Structures 2003; 17:123-144.

12. Luo XY, Calderhead B, Liu HF, Li WG. On the initial configurations of collapsible tube flow. Computers and Structures 2007; 85:977-987.

13. Hazel AL, Heil M. Steady finite-Reynolds-number flows in three-dimensional collapsible tubes. Journal of Fluid Mechanics 2003; 486:79-103.

14. Marzo AXY, Luo XY, Bertram CD. Three-dimensional flow through a thick walled collapsible tube. Journal of Fluids and Structures 2005; 20:817-835.

15. Zhu YF, Luo XY, Ogden RW. Asymmetric bifurcations of thick-walled circular cylindrical elastic tubes under axial loading and external pressure. International Journal of Solids and Structures 2008; 45:3410-3429.

16. Heil M, Waters LS. How rapidly oscillating collapsible tubes extract energy from a viscous mean flow. Journal of Fluid Mechanics 2008; 601:199-227.

17. Luo XY, Cai ZX, LI WG, Pedley TJ. The cascade structure of linear stabilities of flow in collapsible channels. Journal of Fluid Mechanics 2008; 600:45-76.

18. ADINA R\&D Inc. ADINA Theory and Modelling Guide, vols 1-3. ADINA R\&D Inc., 2003.

19. Garbow BS. The QZ algorithm to solve the generalized eigenvalue problem for complex matrices. $A C M$ Transactions on Mathematical Software 1978; 4:404-410.

20. Bathe KJ. Finite Element Procedures. Prentice-Hall: Englewood Cliffs, NJ, 1996.

21. Timoshenko SP, Woinowsky-Krieger S. Theory of Plates and Shells (2nd edn), 1959.

22. Crandall SH, Dahl NC, Lardner TJ. An Introduction to the Mechanics of Solids (2nd edn). McGraw-Hill: New York, 1978. 